\title{
Influencia de las condiciones climáticas de cultivo en la calidad en cosecha y en el comportamiento postcosecha de frutos de Feijoa
}

\section{Influence of weather conditions of crop on harvest quality and post-harvest behavior of pineapple guava fruit}

\author{
Alfonso Parra-Coronado ${ }^{1}$, Gerhard Fischer ${ }^{2}$, \\ Jesús Hernán Camacho-Tamayo ${ }^{3}$
}

Parra-Coronado, A; Fischer, G; Camacho-Tamayo, J. Influencia de las condiciones climáticas de cultivo en la calidad en cosecha y en el comportamiento postcosecha de frutos de Feijoa. Tecnología en Marcha. Vol. 32, Especial. XIII CLIA. Abril 2019. Pág 86-92.

DOI: https://doi.org/10.18845/tm.v32i7.4264

1 Ingeniero Agrícola, Ph.D. Universidad Nacional de Colombia, Facultad de Ingeniería, Departamento de Ingeniería Civil y Agrícola, Bogotá D.C., Colombia. Correo electrónico: aparrac@unal.edu.co

2 Ingeniero Hortícola, Ph.D. Universidad Nacional de Colombia, Facultad de Ciencias Agrarias, Departamento de Agronomía, Bogotá D.C., Colombia. Correo electrónico: gfischer@unal.edu.co

3 Ingeniero Agrícola, Ph.D. Universidad Nacional de Colombia, Facultad de Ingeniería, Departamento de Ingeniería Civil y Agrícola, Bogotá D.C., Colombia. Correo electrónico: jhcamachot@unal.edu.co 


\title{
Palabras clave
}

Acca sellowiana; pérdida de peso; contenido de azúcares; acidez; firmeza.

\section{Resumen}

Las condiciones climáticas de cultivo, que definen las propiedades o atributos de los frutos de feijoa en la recolección, determinan también el comportamiento de los mismos durante la postcosecha. En este estudio se evaluó el efecto de las condiciones climáticas de cultivo en algunos atributos de calidad de la fruta en el momento de la cosecha, así como en su comportamiento durante el periodo postcosecha. Se consideraron dos predios situados en localidades diferentes del departamento de Cundinamarca - Colombia, localizadas a altitudes de 1.800 msnm y 2.580 msnm, en cada una de las cuales se marcaron 20 árboles y se realizó el registró de los parámetros climáticos en el periodo de desarrollo de los frutos hasta la cosecha, para dos periodos diferentes de producción. Se evaluaron periódicamente los parámetros de calidad en el periodo de desarrollo de los frutos hasta la cosecha, así como en el periodo postcosecha. En la postcosecha, los frutos diferenciados por localidad fueron almacenados a temperaturas de $5 \pm 1^{\circ} \mathrm{C}(87 \pm 5 \% \mathrm{HR})$ y $18 \pm 1^{\circ} \mathrm{C}(76 \pm 5 \% \mathrm{HR})$, con valoración de los parámetros de calidad cada 2 días. La conclusión del estudio fue que la calidad de los frutos en la cosecha y durante la postcosecha depende de las condiciones climáticas de cultivo. Los frutos producidos a altitud mayor tienen más sólidos solubles totales y mayor tamaño y peso, presentando en postcosecha menor pérdida de peso y menor firmeza y acidez. A mayor temperatura de almacenamiento los frutos presentaron más pérdida de peso y de firmeza, mayor contenido de azucares y menos durabilidad.

\section{Keywords}

Acca sellowiana; weight loss; sugar content; acidity; firmness.

\begin{abstract}
The weather conditions of crop, which define the properties or attributes of the pineapple aguava (feijoa) fruits at the time of harvest, also determine the behavior of the same during post-harvest. In this study, the effect of the weather conditions of the crop on some fruit quality attributes at the harvest, as well as on its behavior during the post-harvest period, was evaluated. Two farms were considered located in different localities of the department of Cundinamarca - Colombia, located at altitudes of 1,800 masl and 2,580 masl, in each of which 20 trees were marked and the weather conditions registered during the development period of the fruits until the harvest, for two different periods of production. The parameters of quality in the period of development of the fruits until the harvest, as well as during the post-harvest period, were evaluated periodically. In postharvest, fruits differentiated by location were stored at temperatures of $5 \pm 1^{\circ} \mathrm{C}(87 \pm 5 \% \mathrm{RH})$ and $18 \pm 1^{\circ} \mathrm{C}(76 \pm 5 \% \mathrm{RH})$, with assessment of quality parameters every 2 days. The conclusion of the study was that the quality fruit in the harvest and during the post-harvest depends on the weather conditions of crop. Fruits produced at higher altitudes have higher content of total soluble solids, larger size and weight, presenting in post-harvest less weight loss and less firmness and acidity. At higher storage temperature, the fruits presented more weight loss and firmness, higher sugar content and less durability.
\end{abstract}




\section{Introducción}

La feijoa (Acca sellowiana (O. Berg) Burret), se cultiva en Colombia en altitudes que van desde los 1.800 hasta los 2.700 msnm [1]. Una vez cosechadas, las feijoas maduran desde su interior hacia afuera, con cambios en su calidad externa poco significativos después de su recolección, lo cual dificulta la definición del estado de madurez de los frutos por técnicas no destructivas, a la vez que se dificulta realizar propuestas tecnológicas que permitan la conservación de su calidad en postcosecha [1]. El grado de madurez está relacionado directamente con la calidad de los frutos y para su determinación, se hace necesario evaluar diferentes atributos, dentro de los cuales se encuentran la firmeza, el contenido de sólidos solubles totales (SST), la acidez total titulable (ATT) y la relación SST/ATT (RM= Relación de Madurez) [2].

Las condiciones climáticas prevalecientes en el cultivo definen los atributos de calidad de los frutos, con alta influencia de la altitud, la temperatura y la precipitación, que afectan directamente los SST, firmeza y color [3]. En feijoa no se habían realizado investigaciones relacionadas con la incidencia de las condiciones climáticas en los atributos de calidad de los frutos en la cosecha, así como en su comportamiento en postcosecha. Por esta razón, el presente trabajo que es un resumen de los resultados de la tesis doctoral del primer autor [4], tuvo como finalidad estudiar la incidencia de las condiciones climáticas prevalecientes durante el desarrollo de los frutos de feijoa, en algunos atributos de calidad en el momento de la cosecha, así como en su comportamiento postcosecha a dos condiciones diferentes de temperatura de almacenamiento.

\section{Materiales y métodos}

La investigación se llevó a cabo en dos predios ubicados en el departamento de Cundinamarca - Colombia, que cuentan con cultivos de feijoa del clon 41 ('Quimba'), establecidos en el año 2006. Una finca está ubicada en el municipio de Tenjo (451' $23^{\prime \prime} \mathrm{N}$ y $74^{\circ} 6^{\prime}$ ' 33" W) a una altitud media de 2.580 msnm, con condiciones climáticas medias registradas durante la investigación de $12,3^{\circ} \mathrm{C}$ de temperatura, humedad relativa (HR) entre 76,4 y $84,3 \%$ y precipitación media anual entre 190 y $417 \mathrm{~mm}$. La otra finca está ubicada en el municipio de San Francisco de Sales ( $4^{\circ} 57^{\prime} 57^{\prime \prime} \mathrm{N}$ y $74^{\circ} 16^{\prime} 27^{\prime \prime} \mathrm{W}$ ) a una altitud media de $1.800 \mathrm{msnm}$, con condiciones climáticas medias registradas durante la investigación de $18,3^{\circ} \mathrm{C}$ de temperatura, humedad relativa (HR) entre 86,1 y 95,1\% y precipitación media anual entre 573 y $1.400 \mathrm{~mm}$. El manejo del cultivo se realizó de la misma forma en los dos predios, con el fin de descartar el efecto de las variables asociadas al manejo del cultivo.

Mediante estaciones meteorológicas automatizadas iMETOS ECO D2 (Pessl Instruments, Weiz, Austria) dispuestas en cada finca, se obtuvieron los registros meteorológicos cada hora de temperatura $(T)$ y humedad relativa $(\mathrm{HR})$, precipitación acumulada y radiación total diarias, desde plena floración (antesis) hasta la cosecha del fruto, entre los años 2012 a 2014. Se marcaron 10 árboles por parcela experimental y dos parcelas por predio, contando con 40 árboles en total para el estudio. Se marcaron botones florales de aproximadamente $5 \mathrm{~mm}$ de diámetro, con el fin de registrar el desarrollo desde plena floración hasta la recolección de los frutos. Una vez los frutos alcanzaron su madurez fisiológica, se recolectaron 300 frutos en cada finca por dos cosechas. Los frutos fueron separados de acuerdo con el predio de origen para cada cosecha y se almacenaron a temperaturas de $5 \pm 1^{\circ} \mathrm{C}(87 \pm 5 \% \mathrm{HR})$ por un periodo de 31 días y a $18 \pm 1^{\circ} \mathrm{C}(76 \pm 5 \% \mathrm{HR})$ por 11 días.

Las características de calidad medidas durante el desarrollo del fruto fueron tamaño y peso, firmeza, SST y ATT. Durante el periodo de almacenamiento, además de los atributos de calidad anteriores, se midió periódicamente la pérdida de peso (dW) en cinco muestras de dos frutos cada una, utilizando una balanza analítica (aprox. 0,0001 g). La variación de la firmeza se 
determinó mediante el uso de un texturómetro Broofield CT3-4500 (Broofield Engineering, Middleboro, MA, USA) con precisión de $\pm 0,5 \%$, utilizando sonda TA39 y realizándose dos lecturas en cada fruto. La determinación de SST se realizó aplicando la norma NTC 4624 [5] mediante el uso de un refractómetro Eclipse (Bellingham Stanley, Tunbridge Well, UK) con rango de medida de 0 a 32 y precisión de 0,2 ${ }^{\circ}$ Brix. Para la determinación de la ATT se aplicó la norma NTC 4623 [6]. Se determinó además la relación SST/ATT (RM). Para la determinación de las características de calidad durante el desarrollo del fruto, se tomaron muestras al azar semanalmente de un fruto en cada árbol de cada parcela.

En el periodo postcosecha se evaluaron los atributos de calidad cada dos días en los frutos que se almacenaron a las dos temperaturas. Se consideró un diseño estadístico completamente casualizado, con cinco repeticiones por ensayo, tanto para los análisis en precosecha como en postcosecha. El análisis de correlación, análisis de varianza y pruebas de Tukey para los diferentes parámetros de calidad del fruto, para cada finca y para cada cosecha, se realizó mediante el programa estadístico IBM-SPSS v.20 (SPSS Inc., Chicago, IL, USA). Los datos se analizaron mediante estadísticos descriptivos, considerando como factor de dispersión la desviación estándar (DS).

\section{Resultados y discusión}

\section{Desarrollo del fruto (precosecha)}

Crecimiento del fruto

Los frutos producidos en San Francisco de Sales (menor altitud), donde se tienen los registros de mayor T y menor radiación total acumulada (Rad), crecieron con mayor rapidez, con menos días calendario para el desarrollo de los frutos hasta la cosecha, lo cual está acorde con lo reportado para tomate, uchuva, fresas, uvas, mangos, manzanas y ciruelas [4]. Los frutos de mayor peso se obtuvieron bajo condiciones de mayor precipitación total acumulada $(P)$ y mayor HR, lo cual se encuentra dentro de los valores presentados por Parra-Coronado et al. [3] y concuerda con lo reportado para cultivos de granada, tomate y pimientos [4].

Variación de los atributos de calidad medidos

Durante el desarrollo de los frutos, la ATT y los SST mostraron a través del tiempo un comportamiento creciente en sus valores. La variación de SST no fue significativa, presentando

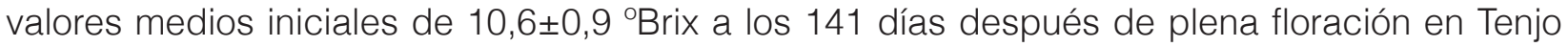
y de 10,8 \pm 0,6 ${ }^{\circ}$ Brix a los 113 días en San Francisco, incrementándose con el crecimiento del fruto, hasta alcanzar en el momento de la cosecha $12,6 \pm 0,8{ }^{\circ}$ Brix en Tenjo y $11,4 \pm 0,8{ }^{\circ}$ Brix en San Francisco. Los valores medios iniciales de la ATT fueron de 1,0 $\pm 0,09 \%$ en Tenjo y de $1,1 \pm 0,07 \%$ en San Francisco, alcanzando en el momento de la cosecha valores de 1,80 \pm 0,11\% en Tenjo y de 1,76 $0,07 \%$ en San Francisco. Este comportamiento de la ATT y los SST durante el crecimiento del fruto concuerda con lo encontrado para frutos de feijoa de los clones 8-4 y 41 [7] y para frutos de guayaba [3].

La RM presentó un comportamiento decreciente con el crecimiento del fruto, lo cual se explica porque la ATT y los SST se incrementan con el crecimiento del fruto, e indica que los SST se translocan a los frutos en una menor proporción que los ácidos orgánicos, que es lo opuesto a lo observado en otros frutos [2]. La relación SST/ATT (RM) presentó valores iniciales de 10,8 1,3

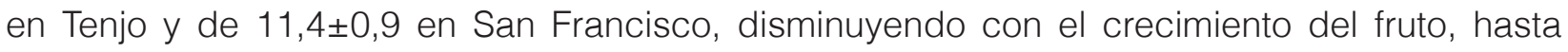

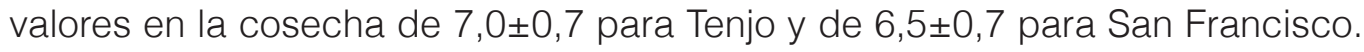


Los frutos presentaron valores medios iniciales de firmeza de $34,0 \pm 6,6 \mathrm{~N}$ en Tenjo y de $30,3 \pm 5$ $\mathrm{N}$ en San Francisco y fue disminuyendo hasta valores de 12,5 $\pm 3,0 \mathrm{~N}$ y de 15,2 $\pm 1,6 \mathrm{~N}$ para Tenjo y San Francisco respectivamente en el momento de la cosecha [3], comportamiento que está acorde con lo encontrado para pera [2]

\section{Almacenamiento del fruto (postcosecha)}

Pérdida de peso $(\mathrm{dW})$ de los frutos en el periodo de almacenamiento

La dW aumenta a través del periodo de almacenamiento a las dos condiciones consideradas, lo cual está acorde con lo reportado para los frutos de feijoa de los clones Quimba y 8-4 [7]. La dW fue menor para los frutos de feijoa almacenados a menor temperatura, comportamiento similar al reportado para peras [2]. La pérdida de peso (dW) de los frutos de feijoa al final del almacenamiento a $18^{\circ} \mathrm{C}$ fue de $13,01 \pm 1,98 \%$ para los provenientes de Tenjo y de $20,37 \pm 1,60 \%$ para los de San Francisco. Los frutos que permanecieron a $5^{\circ} \mathrm{C}$, presentaron valores medios de dW al final del almacenamiento de 5,94 $0,75 \%$ para los provenientes de Tenjo y de $8,48 \pm 1,91 \%$ para los de San Francisco. El análisis de medias indicó diferencias estadísticas en la dW respecto a la temperatura y humedad relativa de almacenamiento, así como respecto al predio de origen. Los frutos almacenados a $18^{\circ} \mathrm{C}$ presentaron diferencias significativas con respecto al lugar de origen y a la cosecha, mientras que los frutos almacenados a $5^{\circ} \mathrm{C}$ no mostraron diferencias.

\section{Variación de los atributos de calidad medidos}

Los frutos de feijoa que permanecieron a $5^{\circ} \mathrm{C}$ durante el almacenamiento, presentaron poca variación en sus contenidos de SST y ATT, así como en su RM, en tanto que los frutos que se mantuvieron a $18^{\circ} \mathrm{C}$ durante el almacenamiento, presentaron incremento en los valores de SST y de RM, así como disminución de ATT a medida que los frutos maduran (figura 1). Los frutos que permanecieron a menor temperatura durante el almacenamiento, presentaron menor cambio en sus atributos de calidad, lo cual se explica porque con el aumento de la temperatura las reacciones enzimáticas aumentan exponencialmente su velocidad [1]. Estos resultados están dentro los rangos reportados por Rodríguez et al. [7] y Velho et al. [8].
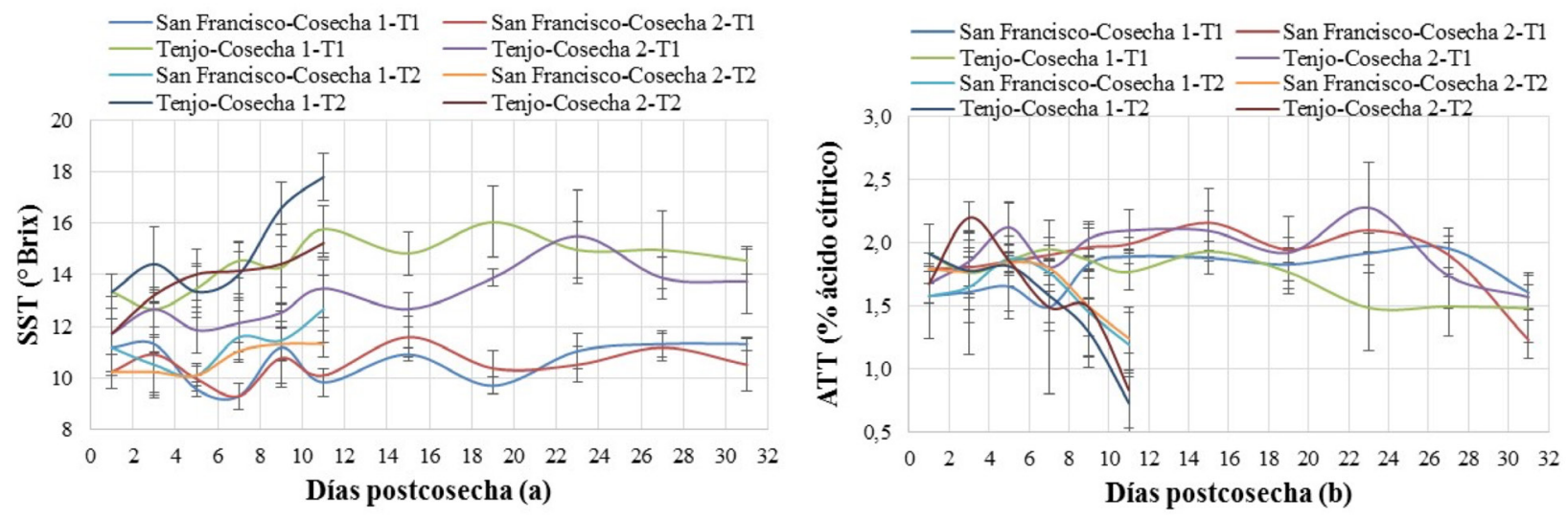

Figura 1. Variación de SST (a) y de ATT (b) de frutos de feijoa (dos cosechas), durante el almacenamiento a $18^{\circ} \mathrm{C}$ (T1) y a $5^{\circ} \mathrm{C}$ (T2). Las barras verticales muestran la desviación estándar. Valores tomados de [4]. 
La firmeza disminuye más rápidamente en los frutos almacenados a mayor temperatura $\left(18^{\circ} \mathrm{C}\right)$, manteniendo un valor aceptable hasta el día 11 de almacenamiento, mientras que los frutos almacenados a $5^{\circ} \mathrm{C}$ la mantuvieron hasta el día 31 de almacenamiento. Al finalizar el almacenamiento a $5^{\circ} \mathrm{C}$, los frutos de San Francisco y de Tenjo presentaron una pérdida de firmeza de 8,2 y $37,1 \%$ respectivamente, mientras que en los frutos que se mantuvieron a $18^{\circ} \mathrm{C}$ las pérdidas de firmeza fueron de 38,5 y $66,0 \%$ para los frutos de San Francisco y de Tenjo, respectivamente. La literatura consultada reporta comportamientos similares para frutos de feijoa de los clones 41 y 8-4 [1], así como para guayabas, champa, arazá y peras [4].

Influencia de las condiciones climáticas

Las feijoas producidas en San Francisco (menor altitud, mayores registros de T y HR, menores registros de Rad), presentaron mayor firmeza y menor SST en el momento de la cosecha y durante el almacenamiento, y mostraron menor contenido de SST, menor RM y mayor ATT y dW, lo cual indica que los predios ubicados en clima frío producen frutos de mejor sabor y con menor dW, que en los predios ubicados en clima más cálido. Estos resultados concuerdan con lo reportado para frutos de ciruela, fresa, mango y manzanas [4]. De igual manera, se reporta que el contenido de SST y ATT, así como el peso, fueron menores para otros frutos cultivados a menor altitud, donde se tienen temperaturas demasiado altas [3]. Las cosechas en las cuales ocurrió la mayor $\mathrm{P}$ y HR, produjeron frutos con menor contenido de SST y menor firmeza, comportamiento que se mantuvo durante el almacenamiento.

Las condiciones climáticas registradas en las dos localidades, no tuvieron influencia sobre los valores de ATT en el momento de la cosecha [4]. El análisis de medias de los resultados del periodo postcosecha, muestra diferencias estadísticas relacionadas con el predio de origen de los frutos y con las condiciones de almacenamiento, para el contenido de SST y ATT, firmeza y RM. La variación de la firmeza, de SST, ATT y RM de los frutos en postcosecha, depende de la temperatura de almacenamiento y de los valores de estos parámetros en la cosecha, valores que se ven afectados por las características climáticas del lugar de producción, prevalecientes en el periodo de desarrollo del fruto.

\section{Conclusiones}

Los frutos más dulces, con mayor pérdida de peso y firmeza y menor durabilidad en postcosecha, fueron los almacenados a mayor temperatura. La altitud de la zona de producción y las características climáticas registradas en el cultivo, mostraron alta influencia en el desarrollo de los frutos de feijoa. La altitud de la zona de producción presentó una relación directa con el tamaño y peso de los frutos en cosecha.

En postcosecha, la temperatura de almacenamiento y el sitio de origen (altitud), presentaron una alta incidencia en la variación de los valores de la firmeza, SST, ATT, RM y dW de los frutos de feijoa, variación que depende de los valores de estos atributos en el momento de la recolección, los cuales están determinados por las características climáticas predominantes en el cultivo durante el periodo de desarrollo del fruto. Se observó que a menor altitud, son menores los SST y la pérdida de firmeza, mientras que es mayor dW y ATT. 


\section{Referencias}

[1] A. Parra C., y G. Fischer, "Maduración y comportamiento poscosecha de la feijoa (Acca sellowiana (O. Berg) Burret). Una revisión", Rev. Colomb. Cienc. Hortic., vol. 7, no. 1, pp. 98-110, 2013

[2] A. Parra-Coronado, J.E. Hernández-Hernández y J.H. Camacho-Tamayo, "Comportamiento fisiológico de la pera variedad Triunfo de Viena (Pyrus Communis L.) durante el período poscosecha", Rev. Bras. Frutic., vol. 28, no. 1, pp. 46-50, 2006

[3] A. Parra-Coronado, G. Fischer and J.H. Camacho-Tamayo, "Development and quality of pineapple guava fruit in two locations with different altitudes in Cundinamarca, Colombia", Bragantia, vol. 74, no. 3, pp. 359-366, 2015

[4] A. Parra-Coronado. "Efecto de las condiciones climáticas en el crecimiento y calidad poscosecha del fruto de la feijoa (Acca sellowiana (O. Berg) Burret)", tesis doctoral, Fac. Agron., Univ. Nal. Colombia., Bogotá, 2014

[5] Jugos de frutas y hortalizas. Determinación del contenido de sólidos solubles. Método refractométrico, Norma técnica colombiana NTC 4624, 1999

[6] Productos de frutas y verduras. Determinación de la acidez titulable, Norma técnica colombiana NTC 4623, 1999

[7] M. Rodríguez, A.E. Arjona y J.A. Gálvis, "Maduración del fruto de feijoa (Acca sellowiana Berg) en los clones 41 (Quimba) y 8-4 a temperatura ambiente en condiciones de la Sabana de Bogotá", Agron. Colomb., vol. 24, no. 1, pp. 68-76, 2006

[8] A.C. Velho et al., "Influência da temperatura de armazenamento na qualidade pós-colheita de goiabas serranas", Rev. Bras. Frutic., vol. 33, no. 1, pp. 14-20, 2011 